\title{
Note: Contrast enhancement and artifact suppression in computed tomography using sinogram normalization
}

Ik-Hwan Kwon, Chung-Ki Hong, and Jun Lim

Citation: Review of Scientific Instruments 89, 016101 (2018);

View online: https://doi.org/10.1063/1.5004061

View Table of Contents: http://aip.scitation.org/toc/rsi/89/1

Published by the American Institute of Physics

\section{Articles you may be interested in}

A transportable Paul-trap for levitation and accurate positioning of micron-scale particles in vacuum for laserplasma experiments

Review of Scientific Instruments 89, 013302 (2018); 10.1063/1.4995955

A novel approach to electron data background treatment in an online wide-angle spectrometer for laseraccelerated ion and electron bunches

Review of Scientific Instruments 89, 013301 (2018); 10.1063/1.5001990

Reflector automatic acquisition and pointing based on auto-collimation theodolite

Review of Scientific Instruments 89, 015101 (2018); 10.1063/1.4986313

On the effect of local barrier height in scanning tunneling microscopy: Measurement methods and control implications

Review of Scientific Instruments 89, 013701 (2018); 10.1063/1.5003851

Hybrid setup for micro- and nano-computed tomography in the hard X-ray range

Review of Scientific Instruments 88, 123702 (2017); 10.1063/1.5011042

Development of sub-100 femtosecond timing and synchronization system

Review of Scientific Instruments 89, 014701 (2018); 10.1063/1.5001768

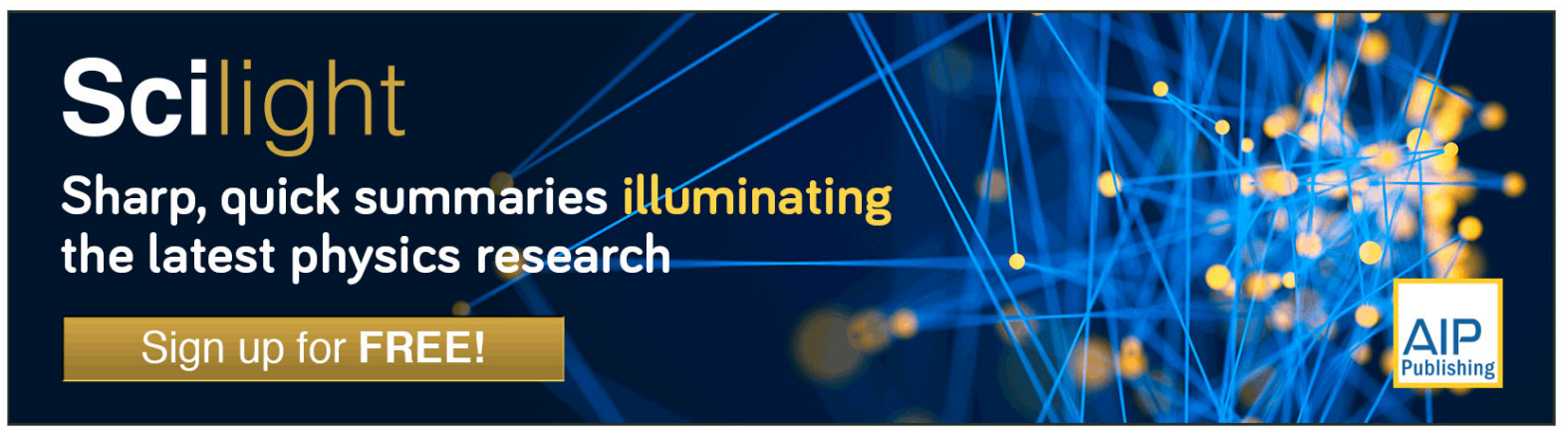




\title{
Note: Contrast enhancement and artifact suppression in computed tomography using sinogram normalization
}

\author{
Ik-Hwan Kwon, ${ }^{1}$ Chung-Ki Hong, ${ }^{1}$ and Jun Lim ${ }^{2, a)}$ \\ ${ }^{1}$ Applied Optics Laboratory, Department of Physics, POSTECH, Pohang 37673, South Korea \\ ${ }^{2}$ Industrial Technology Convergence Center, Pohang Light Source, POSTECH, Pohang 37673, South Korea
}

(Received 11 September 2017; accepted 19 December 2017; published online 3 January 2018)

\begin{abstract}
The intensity and direction of the incident beam at the sample position in synchrotron full-field transmission X-ray microscopy is subject to change. Incident-beam fluctuation in computed tomography results in significant contrast degradation of the reconstructed image. In the present study, we devised a simple method by which that problem could be corrected using sinogram normalization. According to our results, the image contrast was improved by $13 \%$, and the artifacts were suppressed. Published by AIP Publishing. https://doi.org/10.1063/1.5004061
\end{abstract}

Phase-contrast microscopy utilizes the artificial phase delay between the un-diffracted reference wave and the diffracted object wave. It is widely employed not only in optical microscopy but also in synchrotron hard X-ray microscopy. ${ }^{1}$ Synchrotron hard X-ray phase-contrast microscopy takes a series of $2 \mathrm{D}$ radiographs while rotating the sample in order to numerically obtain tomographic slice images by a filtered back-propagation algorithm. ${ }^{2}$ This process is known as X-ray phase-contrast computed tomography (XPCT). XPCT, a nondestructive method for high-resolution investigation of specimen's structural information, is employed in various research fields such as materials science, molecular and biochemical analysis, and others. ${ }^{3}$ Generally, it is assumed that the incident beam is uniform and time-invariant. But if the incident beam is non-uniform and (even) time-varying in intensity and position, every single 2D radiograph must be normalized according to its own background image using the flat-field correction (FFC) method. ${ }^{4,5}$ It is both time-consuming and subject to error. Recently, a method that uses the borders of projections to describe the actual flat-field during a scan in synchrotron computed tomography was proposed. ${ }^{6}$

In this paper, we introduce a simple, sinogram-based method for the correction of incident-beam fluctuation. By this method, tomographic slice-image artifacts are significantly suppressed, and the image contrast is clearly enhanced. A reconstruction software using a similar method is already in use, ${ }^{7}$ but in-depth study on this has not yet been reported.

The projection (or 2D radiograph) of a sample is made up of a set of line integrals at an angle. A set of many such projections under different angles organized in 2D, or in other words, a row of CCD images of different angles, is called a sinogram. This is also known as the Radon transform of the sample. In theory, the inverse Radon transform would yield the original image of the sample. However, if all of the incident beams of 2D radiographs are slightly different, the sinogram contains horizontal stripes [see Fig. 1(1)] that result in contrast degradation of the reconstructed image. To solve this problem, we assume that the incident beam has a constant intensity along

\footnotetext{
a) Author to whom correspondence should be addressed: limjun@postech. ac.kr
}

a row of CCD images. This is a quite reasonable assumption in our case because the effective single pixel size is $\sim 0.45 \mu \mathrm{m}$, the field of view (FOV) is $\sim 50 \mu \mathrm{m}$, and the undulator source is of a long shape in the horizontal direction. Under this assumption, we can define the part where the sample does not exist as the incident beam [for example, the red box in Fig. 1(1)]. Figure 1 shows simulation results for the reconstruction of a SheppLogan phantom. Figures 1(1) and 1(2) show a sinogram and its reconstructed slice for incident-beam intensity variation up to $20 \%$. For the part (red box), we could rescale, for every sinogram, the average intensity of each row so as to equalize it to the average intensity of the first row. Then, we rescaled the entire sinogram according to the scale factors in order to achieve the normalization of the sinogram. In this way, we could obtain (3) the corrected sinogram and (4) its reconstructed slice, which showed high contrast relative to that of Fig. 1(2). Quantitatively speaking, the root mean square (RMS) contrast was increased by $13.6 \%$ (from 0.164 to 0.187 ) and the Michelson contrast (or visibility) by $10.5 \%$ (from 0.160 to 0.176 ). In addition, the streak artifact is effectively diminished. The phantom is a hat shape with a background of 0.2 and a top of 0.3 . However, the reconstructed slice image [Fig. 1(4)] has slightly shifted values ( 0.227 and 0.329 , respectively). The shift is mainly due to the discrete Fourier transformation. It should be noted that the height ( absorption coefficient) of 0.102 is well match with the actual value of 0.1 .

To verify the simulation results, we performed an experiment on the 7C beamline at Pohang Light Source-II (PLS-II). The 7C X-ray Nano Imaging (XNI) beamline is utilized for the purposes of hard X-ray full-field Zernike phase-contrast microscopy. 8,9

It applies off-axis illumination and a hole-type phase plate that provides outstanding contrast enhancement with minimal halo artifact. For imaging, a tungsten objective zone plate of $40 \mathrm{~nm}$ outermost zone width, $150 \mu \mathrm{m}$ diameter, and $0.6 \mu \mathrm{m}$ thickness was used. As a detector, a Scintillator-coupled Optical Microscope (SOM) incorporating thin scintillation crystal (15 $\mu$ m-thick Tb:LSO), a $20 \times$ optical objective, and a largearea CCD with $4096 \times 4096$ sensors of $9 \mu$ m pixel size was utilized. As a sample, a micron-size ruby sphere is used widely for ruby-line spectroscopy. All natural rubies have internal 

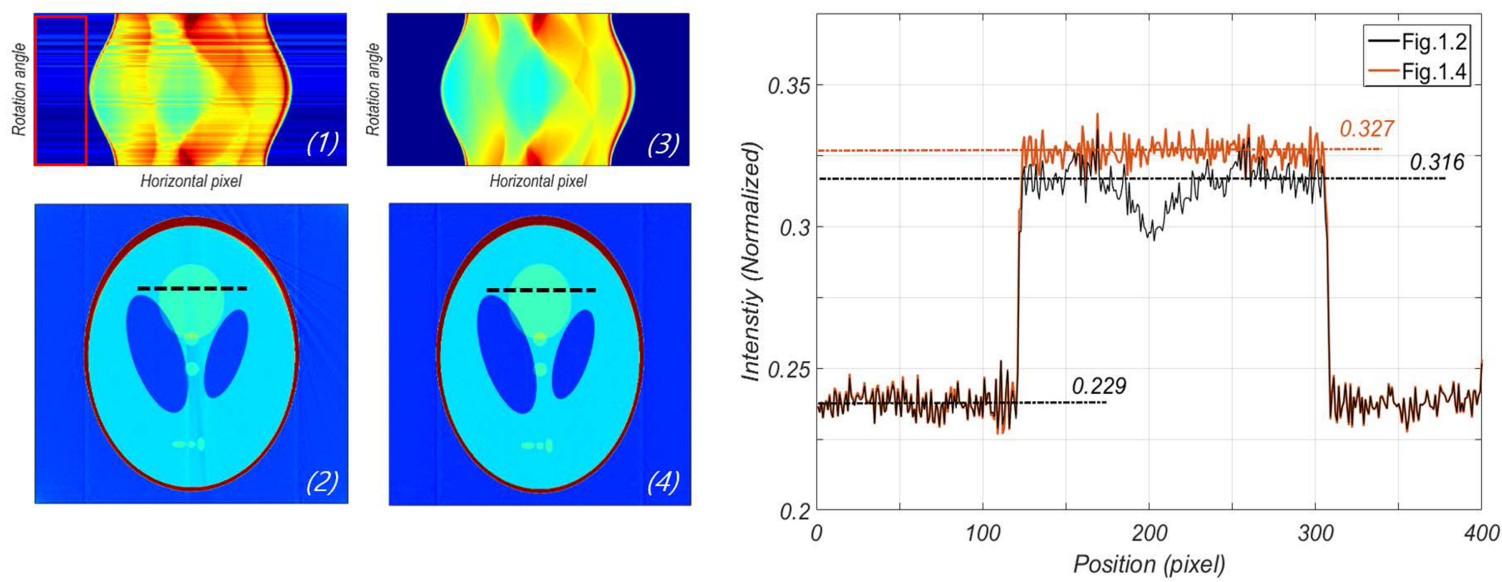

FIG. 1. Simulation results of tomographic reconstruction with the Shepp-Logan phantom. (1) Sinogram for incident-beam fluctuation and (2) its reconstructed slice image. (3) Normalized sinogram and (4) its reconstructed slice image. The line plots show the Michelson contrast of each of the lines of the reconstructed slice images.

defects, including color impurities and inclusions of rutile needles known as silk. The ruby is attached inside a 5- $\mu \mathrm{m}-$ thick thin-glass capillary by electrostatic force. The capillary is mounted on an air-bearing rotation stage (ABRS-150MP; Aerotech). The specifications of the rotation stage are as follows: $<450 \mathrm{~nm}$ radial motion error; $<175 \mathrm{~nm}$ axial motion error; $<10 \mu \mathrm{rad}$ wobble error. In order to obtain a tomographic image of better-than $50 \mathrm{~nm}$ resolution, errors have to be corrected using the intensity summation method. ${ }^{10}$ Figure $2(1)$ shows a transmission X-ray microscopy (TXM) image of a ruby sphere of $\sim 20 \mu \mathrm{m}$ size. There is a very complicated structure inside. A borosilicate glass capillary of $\sim 50 \mu \mathrm{m}$ diameter and $10 \mu \mathrm{m}$ thickness is used to hold the ruby and for rotation error correction. The exposure time of each angle is $5 \mathrm{~s}$ and the lead time is $1 \mathrm{~h}$ for 720 images. Figures 2(2) and 2(3) show the sinograms [of the red line indicated in (1)] before and after correction of the incident-beam fluctuation, respectively. The red box in Fig. 2(2) shows the change in intensity of the incident beam as the ruby rotates. It is a part of the glass capillary, but it is assumed to be empty because the thickness is sufficiently uniform. ${ }^{10}$ The maximum intensity variation was $21.7 \%$ of the first upper line. This indicated

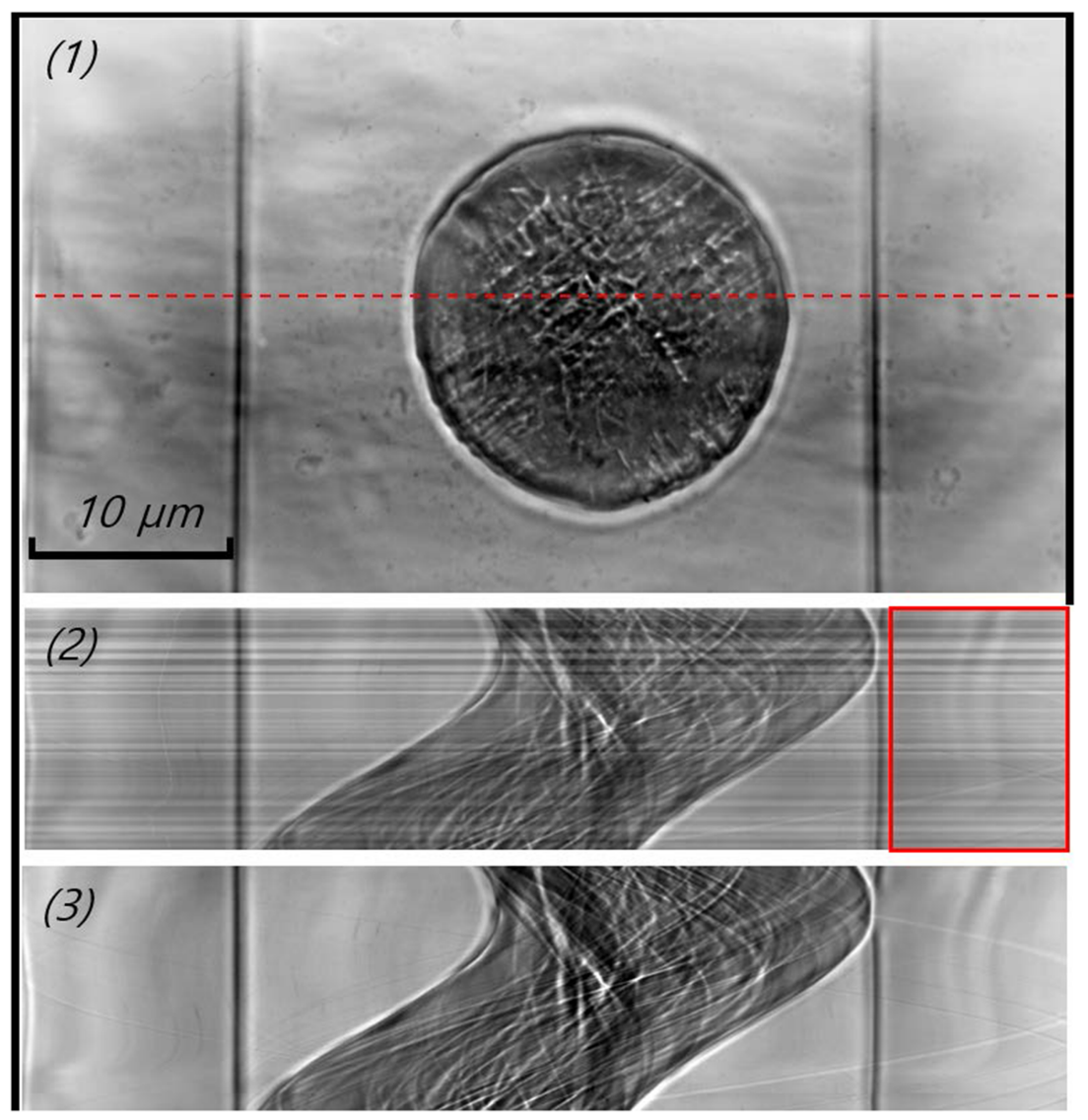

FIG. 2. (1) TXM images of ruby and capillary: sinograms (2) before and (3) after correction of incident-beam fluctuation. 
that the incident beam had changed over time, which phenomenon could be attributed to the beam-direction movement due to electron-beam movement as well as the vibration and thermal instability of the cryogenically cooled double-crystal monochromator and mirrors. In order to stabilize the beam

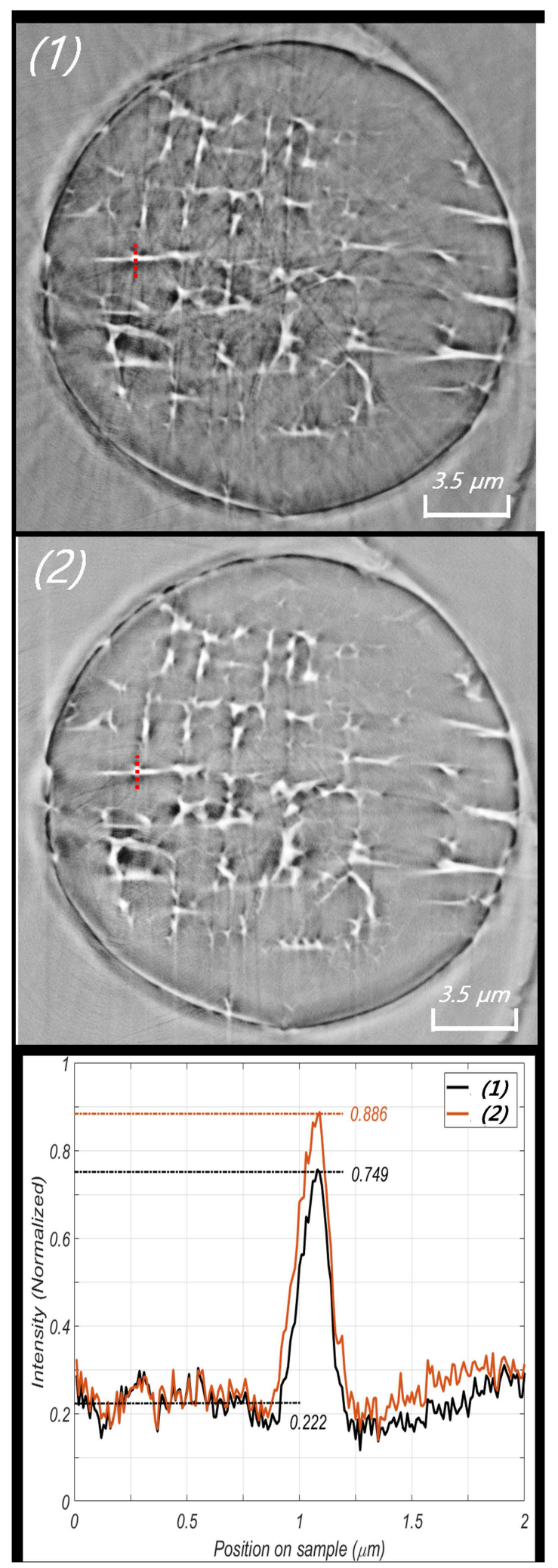

FIG. 3. Reconstructed slice images of ruby powder (a) before and (b) after correction. The line plot of the reconstructed slice image shows a contrast improvement of $10 \%$. movement, diamond screen based x-ray beam monitor and feedback system $(1 \mathrm{~Hz})$ is installed upstream of the sample position. ${ }^{11}$ However, there is high frequency $(>10 \mathrm{~Hz})$ beam motion, which makes intensity fluctuation. As we did in the simulation, after correction of the incident-beam fluctuation, the sinogram had no stripes at all [Fig. 2(3)]. Figures 3(1) and $3(2)$ show reconstructed slice images of the ruby before and after correction, respectively. The corrected image (2) shows higher contrast and prominently fewer artifacts compared with the uncorrected image (1). Quantitatively speaking, the RMS contrast of the images was increased by $13.8 \%$ (from 0.035 to 0.041 ) and the Michelson contrast of the red lines shown in the images was increased by $10.8 \%$ (from 0.530 to 0.588 ). Relative to the simulation results, the absolute amount of RMS contrast was small because of low contrast of X-ray imaging. The DC level shift in the line plot is likely due to improper construction of the ramp filter.

Obviously, there is a constraint of this method in which the sample should be prepared smaller than the FOV because it utilizes the intensity over an empty area that is not covered by the sample. However, if the FOV is larger than the depth of focus (DOF $\sim 4 \Delta \mathrm{r}^{2} / \lambda$ ) of the zone plate, the constraint can be relaxed. In theory, in order to get an isotropic resolution image, the region of interest of a sample should be in the DOF. It means the DOF is the acceptable maximum size of the sample in computed tomography. In this study, all requirements are satisfied: FOV $\sim 60 \mu \mathrm{m}>$ DOF $\sim 35 \mu \mathrm{m}>$ sample $\sim 20 \mu \mathrm{m}$.

In summary, in the synchrotron experimental setup, the intensity and direction of the incident beam are subject to change, which, in computed tomography, causes contrast degradation and artifact generation. In this study, using a simple mode of sinogram normalization, the image contrast was improved, and artifacts were suppressed. Note that this method is effective only to synchrotron-based CT and not for tube X-ray CT. This method of sinogram normalization also can be applied in high-precision 2D-detector-based intensitymeasurement experiments such as those entailing X-ray absorption near-edge spectroscopy (XANES) imaging. ${ }^{12}$

This research was financially supported by grants from the National Research Foundation of Korea (NRF) funded by the Ministry of Science, ICT and Future Planning (Nos. NRF013M2A2A9046502 and NRF-2017R1A2B1002457).

${ }^{1}$ H. S. Youn and S. W. Jung, J. Microsc. 223, 53 (2006).

${ }^{2}$ M. Stampanoni, R. Mokso, F. Marone, J. Vila-Comamala, S. Gorelick, P. Trtik, K. Jefimovs, and C. David, Phys. Rev. B 81, 140105(R) (2010).

${ }^{3}$ A. Sakdinawat and D. Attwood, Nat. Photonics 4, 840 (2010).

${ }^{4}$ V. Titarenko, S. Titarenko, P. J. Withers, F. D. Carloc, and X. Xiaoc, J. Synchrotron Radiat. 17, 689 (2010).

${ }^{5}$ S. E. Park, J. G. Kim, M. A. A. Hegazy, M. H. Cho, and S. Y. Lee, Proc. SPIE 9033, 90335N (2014).

${ }^{6}$ C. Jailin, J. Y. Buffiere, F. Hild, M. Poncelet, and S. Roux, J. Synchrotron Radiat. 24, 220 (2017).

${ }^{7}$ See http://cars9.uchicago.edu/software/idl/tomography.html for information about the reconstruction software.

${ }^{8}$ J. Lim, S. Y. Park, J. Y. Huang, S. M. Han, and H. T. Kim, Rev. Sci. Instrum. 84, 013707 (2013).

${ }^{9}$ J. Lim, H. Kim, and S. Y. Park, J. Synchrotron Radiat. 23, 827 (2014).

${ }^{10}$ I. H. Kwon, J. Lim, and C. K. Hong, J. Synchrotron Radiat. 23, 1237 (2016).

${ }^{11}$ S. Lee, I. H. Kwon, J.-Y. Kim, S.-S. Yang, S. Kang, and J. Lim, J. Synchrotron Radiat. 24, 1276 (2017).

${ }^{12}$ Y. Liu, F. Meirer, P. A. Williams, J. Wang, J. C. Andrews, and P. Pianetta, J. Synchrotron Radiat. 19, 281 (2011). 